\title{
Internacionalización universitaria en contexto de pandemia. Un caso en Chile
}

\section{University internationalization in a pandemic context. A case study in Chile}

Nelson Gutiérrez Lagos ${ }^{1}$, Alixon David Reyes Rodríguez ${ }^{2}$, Marcelo Carvajal Álvarez ${ }^{3}$, Marcelo Carvacho Norambuena ${ }^{4}$

\section{INFORMACIÓN DEL ARTÍCULO \\ Fecha de recepción: 7 de Septiembre de 2021. \\ Fecha de aceptación: 9 de Noviembre de 2021.}

$\overline{1}$ Doctor en Educación, Universidad de Concepción. Docente-investigador, Universidad Adventista de Chile Chile.

E-mail: nelsongutierrez@unach.cl Código ORCID:

https://orcid.org/0000-0001-9227-652X

\footnotetext{
2 Doctor en Educación, Universidad Pedagógica Experimental Libertador. Docente-investigador, Universidad Adventista de Chile - Chile.

E-mail: alixdavid79@gmail.com

Código ORCID:

https://orcid.org/0000-0001-9857-0930
}

${ }^{3}$ Magíster en Educación, Universidad de Chile. Docente-investigador, Universidad Adventista de Chile Chile.

E-mail: marcelocarvajal@unach.cl

Código ORCID

https://orcid.org/0000-0002-4743-2906

4 Estudiante de Ingeniería Civil Informática, Universidad Adventista de Chile. Investigador, Universidad Adventista de Chile - Chile.

E-mail: informaticodd@unach.cl

Código ORCID:

https://orcid.org/0000-0003-3464-3488

CITACIÓN: Gutiérrez, N., Reyes, A., Carvajal, M., \& Carvacho, M. (2021). Internacionalización universitaria en contexto de pandemia. Un caso en Chile. Podium, 40, 105-124. doi:10.31095/podium.2021.40.7

\section{ENLACE DOI:}

http://dx.doi.org/10.31095/podium.202 1.40 .7

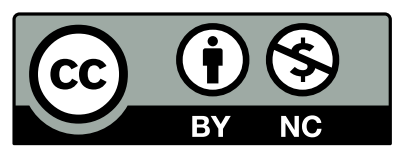

\section{Resumen}

Este trabajo analiza los procesos de internacionalización de la Universidad Adventista de Chile, con énfasis en período de pandemia. La investigación tiene un enfoque cuantitativo, descriptivo y diseño transversal. La muestra fue intencional con 288 informantes. Se aplicó un cuestionario, y los resultados resaltan el crecimiento de las instancias de internacionalización desde 2019, superando en el año 2020 la participación de personas ligadas a la institución aprovechando la virtualidad en un contexto de pandemia. Sobre el $50 \%$ de las instancias de internacionalización se dan por convenios, evidenciado que hay un umbral de relaciones institucionales que pueden consolidarse a partir de convenios entre universidades e instituciones. Si bien, la internacionalización ha implicado relaciones en varios continentes, los países latinoamericanos son los que, con mayores instancias se ha logrado de participación. La pandemia ha advertido en la virtualidad una alternativa para generar, incrementar y consolidar las instancias de internacionalización universitaria.

\section{Palabras Clave:}

Universidad, internacionalización, intercambio, movilidad académica, virtualidad, pandemia.

Clasificación JEL: I21, OO3.

\section{Abstract}

This paper analyzes the internationalization processes of the Adventist University of Chile, with emphasis on the pandemic period. The research has a quantitative, descriptive and cross-sectional design. The sample was intentional with 288 informants. A questionnaire was applied, and the results highlight the growth of internationalization instances since 2019, exceeding the participation of people linked to the institution taking advantage of virtuality in a pandemic context in 2020 . Over $50 \%$ of the internationalization instances are given by agreements, evidencing that there is a threshold of institutional relationships that can be consolidated from agreements between universities and institutions. Although internationalization has involved relations in several continents, Latin American countries are the ones that have been able to participate in the greatest number of instances of internationalization. The pandemic has found in virtuality an alternative to generate, increase and consolidate the instances of university internationalization.

Keywords:

University, internationalization, exchange, academic mobility, virtuality, pandemic. JEL Classification: I21, OO3.

$$
105
$$

PODIUM No. 40, Diciembre 2021, pp. 105-124

(C) Universidad Espíritu Santo - UEES

ISSN: 1390-5473 e-ISSN: 2588-0969 


\section{Introducción}

Si bien es cierto, los procesos de internacionalización universitaria se manifiestan con el nacimiento de la universidad (García, 2005), puede decirse que los procesos de internacionalización inician en América Latina a partir del legado que deja la Reforma de Córdoba (Argentina) en 1918, a partir de dos conceptos que aparecen vinculantes como son, los de integración y autonomía universitaria (Abba y Streck, 2021). Por supuesto, debe reconocerse que la región heredó el modelo anglosajón de internacionalización universitaria, encontrando redefiniciones, acomodos y varianzas según los modelos educativos de los Estados nacionales latinoamericanos (Sebastián, 2017). Durante todo el siglo $\mathrm{XX}$, ambos conceptos, integración $\mathrm{y}$ autonomía universitaria, han sido centrales en los procesos de desarrollo, fortalecimiento y consolidación de la institución universitaria latinoamericana. Así, la internacionalización ha pasado a convertirse de un evento extraordinario y destacable (Santos y Román, 2003), a un proceso imperativo e imprescindible (León y Madera, 2016), y lo es, no en la medida de una moda o tendencia, sino en la medida en que las instituciones de educación universitaria asumen procesos que tienden a fortalecer sus propuestas formativas en los ámbitos de la docencia, la investigación, la vinculación con el medio y transferencia tecnológica, a través de la interacción y el diálogo académico-científico, además de los procesos de gestión al compartir experiencias y tener patrones de colaboración y comparación con sus correlatos institucionales en otros países. Se sabe que en Chile, este proceso de internacionalización inicia por el año 1950, sin embargo, cobra fuerza entrada la década de los '90 del siglo XX a partir de iniciativas estatales (Latorre y Ramírez, 2021), comprendiendo, claro está, el modelo político-económico de Chile que tiene su correlato en el sistema educativo chileno (Mönckeberg, 2014; Villalobos y Quaresma, 2015).

El desarrollo científico y el avance de la educación, además del no menos visible proceso de globalización (Kotorov, Krasylnykova, Zhdanov, y Mazarra 2020), han fungido como marco en el que la internacionalización universitaria pasó a ser una tarea ineludible, necesaria en todo proceso de gestión (Escudero, 2010), ya no sólo institucional, sino también a nivel de política pública en los Estados nacionales, es decir, la internacionalización es precisada ahora como necesidad y como requerimiento de sus instancias superiores en educación (Latorre y Ramírez, 2021; Ramírez-Valdivia, Latorre y Bustos, 2020; Sebastián, 2005).

Generalmente, una institución universitaria apunta a la internacionalización para generar redes de colaboración e integración que ayuden al fortalecimiento de la misión institucional, alcanzando intercambios académicos y pasantías (estudiantes, docentes, otros funcionarios), homologación de estudios, proyectos conjuntos de investigación, desarrollo y posicionamiento de programas académicos conjuntos en pregrado y posgrado, co-tutelas y co-tutorías, celebración 
conjunta de actividades de extensión como congresos y similares, actividades de innovación y transferencia de conocimiento, proyección de un perfil internacional, posicionamiento y visibilidad (Gómez, 2020), innovación educativa (Llano, Carlozama, Tipán, y Tipán 2021), consolidación de una concepción global en la formación universitaria e intercultural, acceso a fondos internacionales (Latorre y Ramírez, 2021), entre otras acciones. Como ya se ha destacado, esto permite visualizar concepciones, modelos $\mathrm{y}$ políticas que enriquecen las propuestas formativas propias de la institución, además que permite generar posibilidades de doble titulación y el reconocimiento de los sistemas crediticios en casos singulares atendiendo a perspectivas profesionales que atiendan al fenómeno de la globalización y la internacionalización.

El proceso de internacionalización puede darse a través de: convenios interinstitucionales, redes de universidades, núcleos de investigación, o simplemente, mediante instancias en las que, sin necesidad de convenios, los académicos, estudiantes y funcionarios de la institución, participan en actividades tributantes a los fines de la universidad, sean, congresos, redes o grupos de investigación, mesas técnicas, asesorías y/o consultorías interinstitucionales y/o gubernamentales foráneas, entre otros. Siendo así, este trabajo tiene como propósito analizar el proceso de internacionalización de la Universidad Adventista de UnACh en el contexto de la pandemia.

\section{Revisión de literatura}

Santos y Román (2003) sistematizan el proceso de evolución de la internacionalización de la Facultad de Ciencias de la Conducta de la Universidad Autónoma del Estado de México. Entre las conclusiones de este trabajo se destaca la noción de internacionalización que asume a posteriori la institución. Allí declaran que la internacionalización "no consiste solamente en un intercambio de estudiantes y doctores" (Santos y Román, 2003: 18), sino que tiene que ver con un proceso de mayor complejidad y que incorpora la visión en red de la educación universitaria en un contexto planetario, o como lo mencionara Albornoz (2013, 2018), se trata de un proceso que ocasiona el necesario derrumbamiento de la noción del quehacer aldeano y el provincianismo institucional. Estos señalamientos son relevantes, dado que en un estudio conjunto realizado por la Organización de las Naciones Unidas para la Educación, la Ciencia y la Cultura - UNESCO y el Instituto Internacional para la Educación Superior en América Latina y el Caribe IESALC (2020), se advierte que la gran afectación en los programas de internacionalización universitaria se ubica precisamente en la movilidad estudiantil, de los docentes e investigadores, acentuándose esto mucho más en tiempos de pandemia, tiempos en los que la aplicación de medidas sanitarias como el confinamiento escalado, el cierre de aeropuertos e instituciones, dificulta esta alternativa de internacionalización.

Munadi (2020) expone la experiencia de la Universidad Estatal Islámica de 
Yogyakarta y la Universidad Estatal Islámica de Malang, en la que destaca que, la internacionalización en ambas instituciones ha sido posible a partir de una planificación estratégica que ha apuntado a la inserción de estas instituciones en el espectro de la comunidad académica internacional. Y ello, habida cuenta de la contracción simbólica de instituciones islámicas a propósito de los prejuicios de índole ideológico, político y religioso (Bermejo, 2016; Grosfoguel, 2014). A su vez, Bruhn (2020), destaca que la internacionalización es imprescindible en los contextos universitarios, y que ello no puede alcanzarse si no hay una implicación de las tecnologías de información y comunicación, lo que ha quedado en evidencia en el período inaugurado a partir del inicio de la pandemia por SARS-COV-2 (causante de la enfermedad del Coronavirus en adelante Covid-19).

Taşçi (2021), presentó un estudio realizado a propósito del impacto del Covid-19 en los procesos de internacionalización en el contexto universitario, en el cual concluye que el proceso más afectado en estas instancias, es la movilidad académica de los estudiantes. De hecho, sostiene que esta pandemia ha generado una ampliación de las brechas socioeconómicas de los estudiantes, afectando a los de mayor vulnerabilidad económica, por lo que, las instituciones de educación universitaria tendrán que disponer de nuevos mecanismos para no ralentizar los procesos de internacionalización en esta población. Una de sus propuestas radica en la virtualidad como manifestación de voluntad político-económica institucional, en las áreas en las que se pueda materializar por esta vía el intercambio académico y la movilidad virtual.

En el marco de investigaciones que consideran el tema de la internacionalización en tiempos de pandemia, especialmente en América Latina, Gacel-Ávila (2020) sostiene que esta última representa un riesgo mayor para este proceso, y afirma que el desafío más importante está representado en la movilidad física de estudiantes, profesores e investigadores. A esto agrega que, la dificultad para mantener la matrícula en los próximos años tendrá implicaciones financieras para las instituciones y deberán redistribuir presupuestos, afectando posiblemente procesos asociados a internacionalización restringiendo aún más las posibilidades de quienes puedan optar a los beneficios de este proceso. En este mismo orden de ideas se presenta la investigación de Parker y López (2020), quienes analizan las consecuencias de la pandemia en el proceso de internacionalización, partiendo de la experiencia de la Universidad de Santiago de Chile. Estos investigadores advierten las mismas complicaciones de las que ya habla Gacel-Ávila (2020). Y a esto suman: "La internacionalización de la Educación Superior (sic) adoptará nuevas formas totalmente virtuales o mixtas en detrimento de lo presencial" (p. 16). Asimismo, Gómez (2020) en su trabajo apunta al desarrollo sostenido y progresivo de instancias virtuales como mecanismo de reorientación de políticas 
asociadas a la internacionalización dadas las condiciones impuestas por la pandemia y las secuelas financieras en las instituciones universitarias que implican la priorización presupuestaria. A esto es a lo que le denominan Fairlie, Portocarrero y Herrera (2021), internacionalización en casa.

Vale la pena atender procesos de instituciones que, entendiendo el panorama de ralentización que está causando la pandemia, vienen generando cambios importantes en sus políticas endógenas de internacionalización. Por ejemplo: la Universidad Nacional Autónoma de Honduras-UNAH (2020), ha empleado protocolos en el contexto de la internacionalización atendiendo a patrones de seguridad y modificación de la gestión considerando la pandemia por Covid-19, quienes al igual que lo planteado por Parker y López (2020) desde la Universidad de Santiago de Chile, confirman la necesidad de generar mayor aproximación virtual o híbrida, dadas las circunstancias. Pero, incluso, proyectan que, aún después de la pandemia, estas condiciones de trabajo colaborativo e intercambio académico, podrán mantenerse en disminución de estancias presenciales.

En este orden de ideas, y comprendiendo que, si bien es cierto, la pandemia ha sido devastadora al considerar la expansión de los contagios y muertes, el colapso de los sistemas sanitarios, el derrumbe económico, el deterioro de las condiciones socioeconómicas de las poblaciones más vulnerables, la ampliación de brechas (Díaz-Castrillón y
Toro-Montoya, 2020; Giroux, 2020; Murillo y Duk, 2020; Parker y López, 2020), las instituciones universitarias han tenido que ir haciendo adecuaciones a fin de poder atender al estudiantado y seguir ofreciendo sus proyectos de formación profesional. Entendiendo que estos fenómenos se hacen centrales en la actualidad para el potenciamiento de este tipo de instituciones, la Universidad Adventista de Chile (UnACh) ha ido generando en el tiempo una serie de mecanismos y procedimientos para concretar procesos de internacionalización tributantes al desarrollo de sus propuestas formativas en el marco de su modelo educativo (Dirección de Docencia, 2021), y más ahora, atendiendo al contexto de pandemia.

Es de considerar que la UnACh es una institución de carácter privado $\mathrm{y}$ confesional, registrada e inscrita en el Ministerio de Educación de Chile, con reconocimiento oficial desde 1990, y que participa de una red de instituciones universitarias adventistas a nivel mundial, siendo una de las 113 instituciones conformantes (Universidad Adventista de Chile, 2018). Además de ello, la UnACh es la única universidad regional en la Región de Ñuble (Maas, 2018), habida cuenta de que la presencia de otras instituciones en la región, estriba en el posicionamiento de sedes emergentes. Así, la UnACh presenta una propuesta formativa que implica cinco facultades: Facultad de Educación y Ciencias Sociales, Facultad de Ciencias de la Salud, Facultad de Ciencias Jurídicas y Sociales, Facultad de Teología y la Facultad de Ingeniería y Negocios. 
Ofrece, a la fecha, 28 carreras de pregrado y siete programas de posgrado (Magíster en Educación; Magíster en Psicología; Magíster en Misión; Magíster en Salud Pública; Magíster en Ciencias de la Motricidad Humana; Magíster en Fruticultura Moderna; Magíster en Gestión de Proyectos de Innovación Social), ofertas de diplomaturas y cursos varios no conducentes a título.

Tal y como ha sucedido con todas las universidades en Chile, la UnACh, tuvo que hacer los ajustes necesarios a fin de atender sus procesos académicos $\mathrm{y}$ administrativos a partir de la mixtura entre la presencialidad y la virtualidad, considerando todas sus implicaciones, es decir: educación a través de plataformas virtuales, adecuación de protocolos didácticos, capacitación de funcionarios, profesores y estudiantes, inversiones para las necesarias adecuaciones tecnológicas, adquisición de software, equipamiento de aulas, entre muchas otras cosas (Ministerio de Educación, 2020; Ordorika, 2020). La internacionalización fue uno de los procesos transversalizado por alternativas y mecanismos de atención. Siendo así, y en el tenor de este tema surgen preguntas a considerar: ¿cómo ha evolucionado este proceso en la UnACh en términos de participación?, ¿qué implicaciones ha tenido en cuanto al aumento o la disminución de instancias de internacionalización?, ¿cómo ha sido el comportamiento de la UnACh y sus diferentes unidades en procesos de internacionalización en tiempos de pandemia, considerando convenios $\mathrm{u}$ otras posibilidades de interacción?, ¿hay proyecciones en internacionalización?, ¿cuáles son las modalidades más vinculadas en los últimos años, y en especial a partir de la pandemia?

\section{Metodología}

Este trabajo tiene un enfoque cuantitativo, de carácter descriptivo y un diseño transversal. Se diseñó y aplicó un cuestionario ad hoc para el estudio de acuerdo con los objetivos de esta pesquisa, con procesos de validación de coherencia y consistencia interna por pares expertos, y una prueba piloto. El instrumento se aplicó a fines de 2020 y consideró la participación en instancias de internacionalización por parte de docentes, estudiantes, administrativos y otros miembros de la universidad, tomando en cuenta la etapa comprendida entre 2017 y 2020, con especial incidencia en el año 2020, debido al contexto de pandemia. El muestreo con el que se trabajó fue intencional dadas las características del estudio (Otzen y Manterola, 2017) y las características de las unidades muestrales. El instrumento empleado no era de llenado obligatorio. Así, la cantidad total de informantes ascendió a 288. Se firmó un consentimiento informado vía digital inserto en el cuestionario. Se aplicó el análisis estadístico descriptivo utilizando la herramienta de Excel.

\section{Resultados}

Dado que el propósito de la investigación apunta a analizar el proceso de internacionalización de la UnACh, contextualizando el tiempo de pandemia, vale la pena considerar algunos 
resultados de interés afines a este enunciado. Así, los resultados de esta pesquisa declaran que un $74 \%$ de los informantes manifiesta haber participado en instancias de internacionalización en el período en estudio, o sea, entre 2017 y 2020. En la Figura 1 se aprecia Si bien es cierto que hay decrecimiento en el año 2018, ya para 2019 se produce un incremento en número de instancias de internacionalización. Sin embargo, durante 2020, la cantidad se duplica llegando a un $59 \%$ de las actividades reportadas en este año, mucho de esto aderezado por la oportunidad que ha brindado la virtualidad.

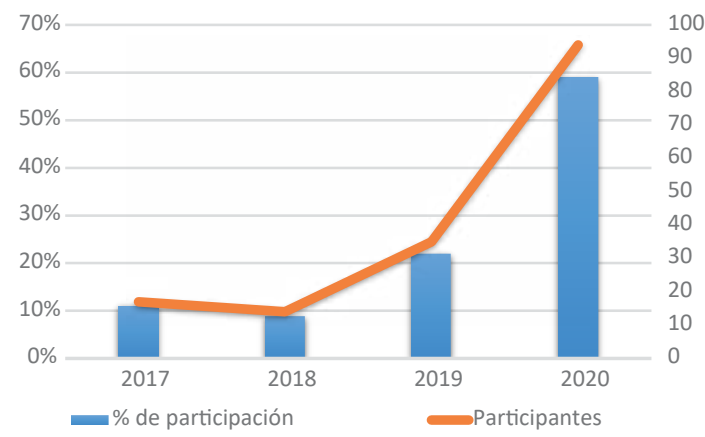

Figura 1. Relación de participación en instancias de internacionalización 2017-2020.

Fuente: Dirección de Docencia UnACh.

De hecho, las medidas de cuarentena y confinamiento inician en Chile en marzo de 2020, y hasta la fecha sólo se había realizado el 6\% de las instancias de internacionalización (3\% en enero 2020 , $3 \%$ en febrero 2020 ), destacando que el
$94 \%$ restante fue realizado entre marzo y diciembre de 2020 en un contexto de pandemia y confinamiento, teniendo los meses de septiembre, octubre y noviembre como los de mayor ocurrencia, y el máximo de 29 actividades de internacionalización en octubre de 2020.

La participación en instancias de internacionalización se diversifica entre tanto hay docentes, estudiantes, administrativos y otros funcionarios de la universidad, siendo los docentes (54\%) quienes cuentan con mayor cantidad de participación en este proceso (Ver Figura 2).

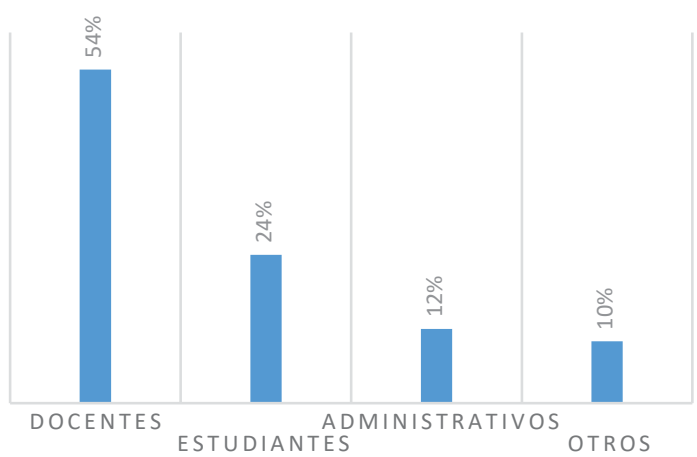

Figura 2. Caracterización de participantes en instancias de internacionalización 2017-2020.

Fuente: Dirección de Docencia UnACh.

En el periodo 2017-2020 se realizaron 168 actividades en función de la internacionalización (Ver Tabla 1), correspondiendo el $31 \%$ a Otros, lo que representa una debilidad en tanto no pueden ser adjudicadas a alguna unidad

Tabla 1 .

Unidades participantes en instancias de internacionalización 2017-2020.

\begin{tabular}{lc}
\hline Unidad & Número de instancias \\
\hline Facultad de Ingeniería y Negocios-FAIN & 35 \\
Facultad de Educación y Ciencias Sociales- FECS & 33 \\
Facultad de Teología & 18 \\
Dirección de Postgrado & 10 \\
Otras unidades & 21 \\
Otros & 51 \\
\hline
\end{tabular}

Fuente: Dirección de Docencia UnACh. 
en particular. Mientras que, entre las unidades declaradas, las que más dinamismo tuvieron fueron FAIN (21\%) y FECS (20\%).

Prácticamente la mitad de las acciones tributantes a la internacionalización se han realizado en el marco de convenios firmados por la universidad (51\%). Esto se explica también en el contexto de dos dimensiones a considerar: 1) la participación en congresos científicos, jornadas, seminarios, proyectos y grupos de investigación, y similares, han sido declaradas como actividades de internacionalización, y estas se realizan sin la necesidad de tener convenios firmados para su desarrollo; 2) se han desarrollado actividades que, si bien es cierto pueden ser desarrolladas en el marco de convenios, se han adelantado, dado que estos procesos de firmas pueden ralentizar el avance de ciertas actividades. El hecho de que cerca del $50 \%$ de las actividades realizadas se hayan desarrollado sin convenios de por medio, implica también un umbral de posibilidades que puede cubrirse a partir de dichas actividades en el contexto de convenios que se firmen, a saber, congresos interinstitucionales, proyectos, grupos de investigación, publicaciones conjuntas, fondos de investigación, pasantías, movilidad académica, entre otros.

Las actividades realizadas han sido desarrolladas en su gran mayoría en la modalidad virtual (62\%), y esta información es relevante dado que la mayoría de estas actividades se han generado a partir de marzo de 2020 en el contexto de la pandemia y el confinamiento en Chile. Dentro de las actividades de internacionalización que se han realizado en modalidad virtual están: aulas espejo, eventos académico -científicos (congresos, jornadas de investigación, conversatorios y similares), webinarios, movilidad digital, encuentros virtuales, videoconferencias, Casa Abierta digital, proyectos de investigación en forma colaborativa, participación en grupos y redes de investigación, entre otros.

Con respecto al tipo de actividades presenciales realizadas en el marco de actividades de internacionalización (Ver Figura 3), se tiene que la participación en

Figura 3. Tipo de actividad (modalidad presencial).

Fuente: Dirección de Docencia, UnACh.

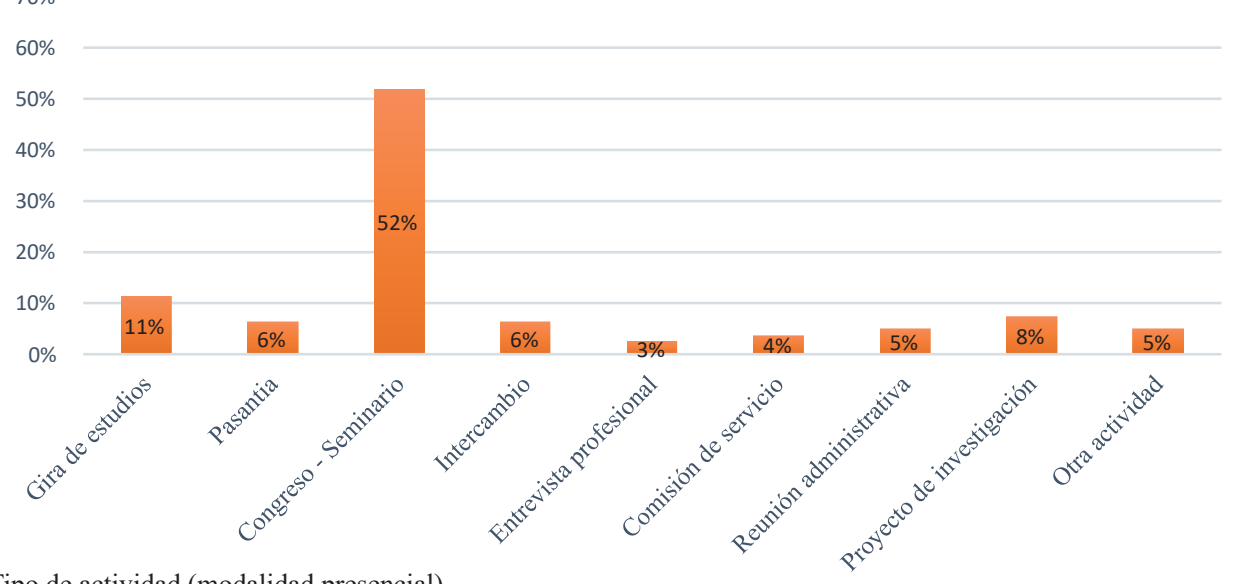


congresos y seminarios ha sido la de mayor ocurrencia (52\%). Llama la atención que, actividades como las pasantías, los intercambios (movilidad académica), y las comisiones de servicio, que son actividades proclives a convenios académicos, registraron bajas tasas.

Entre las actividades virtuales realizadas (Ver Figura 4), se tiene que las videoconferencias (congresos, seminarios, entre otros) han sido las más frecuentes. No obstante, se registró mayor diversidad y participación en las actividades, asunto que puede deberse a las facilidades de la modalidad virtual.

En la Figura 5 se presenta el conjunto de acciones realizadas durante el cuatrienio en consideración, destacando los proyectos de colaboración académica. La mayoría de estas acciones se han generado en contexto de pandemia, y, por tanto, en el ámbito de la virtualidad, con una tasa de $76 \%$ de previsión de continuidad.

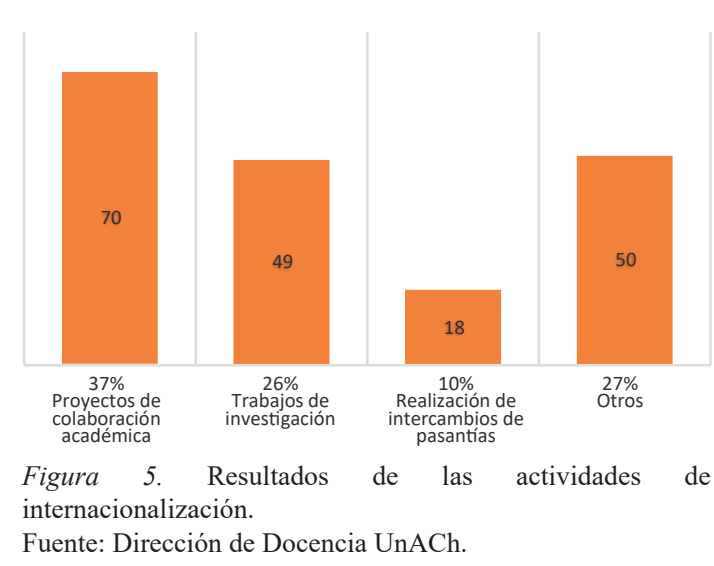

La participación en instancias de internacionalización ha permitido a la comunidad académica de la UnACh, generar articulación con instituciones de una diversidad de países en América, Europa y África. Los países con los que se ha generado mayor articulación son: Brasil (20\%), España (17\%) y Colombia (16\%), seguidos de cerca por otros países como: Perú (10\%), Argentina (9\%), México (8\%), Estados Unidos (6\%), y Ecuador (5\%). Se destaca, además que las instituciones con las que se ha generado alguna vinculación académicoadministrativa, han sido instituciones del

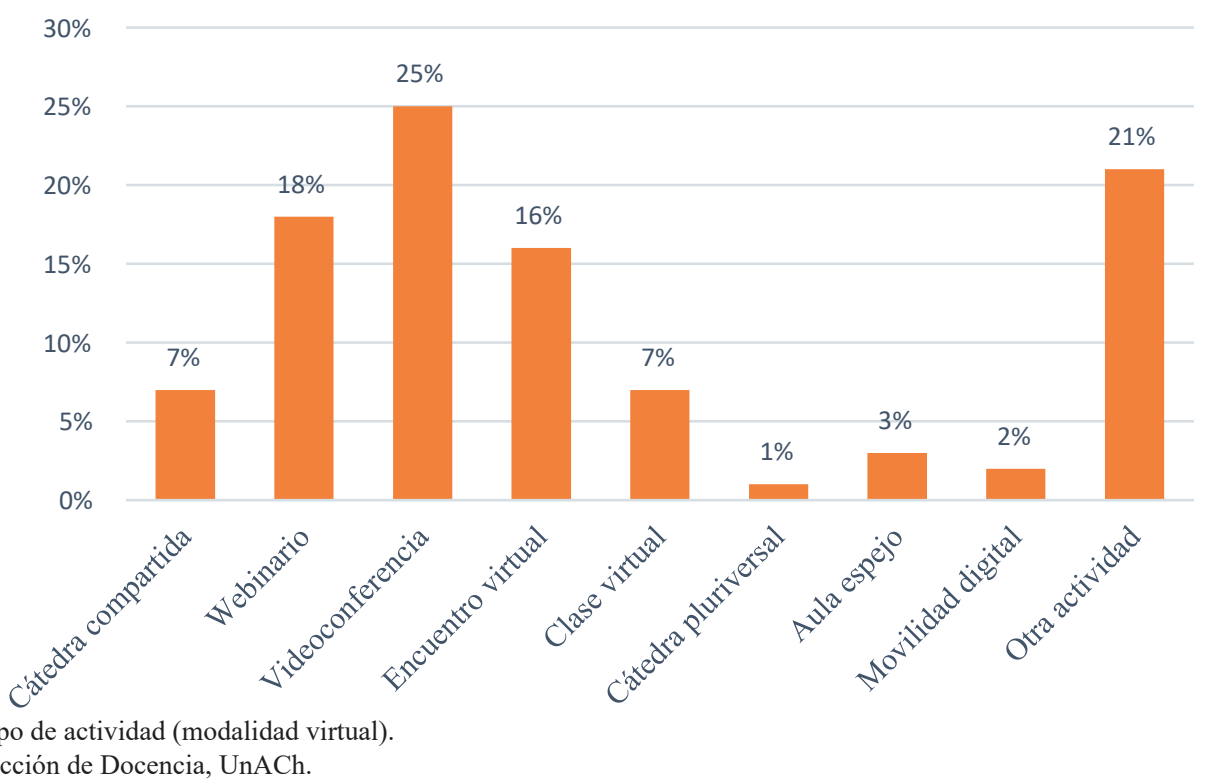

Figura 4. Tipo de actividad (modalidad virtual) Fuente: Dirección de Docencia, UnACh. 
sistema educacional adventista como del sistema educacional no confesional a nivel internacional. Entre algunos de los casos más relevantes están: la Universidad Peruana Unión, la Universidad de Montemorelos, la Universidad Adventista de Sao Paulo, la Universidad de Cundinamarca, Andrews University, entre otras.

Tal y como se ha destacado desde el principio, la internacionalización como proceso en el contexto universitario ha pasado a convertirse en un imperativo, a decir de León y Madera (2016), por lo que, los esfuerzos de las instituciones universitarias deben avanzar hacia estos derroteros. De esta forma también lo destacan Quiroga y Trujillo (2020), en una investigación sobre la internacionalización en América Latina, esto es, que se trata de un proceso que ya no sólo puede ser concebido como apetecible, sino como uno ineludible que da cuenta de los desafíos de la educación universitaria a nivel global y la importancia que viene cobrando a nivel de las políticas públicas (De Wit y Hunter, 2015). Por supuesto, mucho de esto viene aderezado por la necesidad de generar políticas de articulación que permitan la movilidad académica, tanto de estudiantes como de profesores. No obstante, este tipo de políticas también se encuentra inserta en el marco de un debate relevante sobre la polarización que genera en torno a tres aspectos fundamentales: a) por un lado, la necesidad de ampliar la concepción de internacionalización que la reduce a procesos de movilidad estudiantil y profesoral. De hecho, este último aspecto representa una tendencia en procesos de internacionalización en algunas sociedades, como por ejemplo, en Chile, dado que en las universidades conformantes del Consejo de Rectores de Universidades Chilenas (CRUCH), la movilidad de académicos y estudiantes se convierte en la principal y mayoritaria instancia de internacionalización (CRUCH, 2020; Latorre y Ramírez, 2021). Lo mismo sucede en Canadá, donde, según un estudio realizado por Buckner, Clerk, Marroquín \& Zhang (2020), en 32 experiencias de internacionalización analizadas en Canadá, la mayor incidencia reside justamente en la movilidad de estudiantes y académicos; b) por otro lado, y en segundo lugar, se encuentra la dependencia o el anclaje de este tipo de políticas a lógicas de mercado basadas en tasas de movilidad y empleabilidad, que de alguna forma tienden a procesos de homogeneización de las mallas curriculares y el discurso del aseguramiento de la calidad bajo patrones que unifican horizontes de formación (Caamaño y Chacón, 2017; Cancino, 2010; Lemaitre, 2005); y, c) la necesidad de pensar la educación desde la condición humana y la conformación del sujeto, además de la necesaria contextualización de los procesos de formación (Reyes, 2016).

En este contexto, por una parte se advierte que la globalización ha empujado a las instituciones universitarias a generar una apertura en el contexto internacional, y por otra parte se apunta a pensar en procesos que, si bien es cierto, no pueden deslindarse del contexto internacional, apuntan más hacia un 
desarrollo de lo local en un marco de reconocimiento de lo nacional e internacional y viceversa, consustanciado además, con un preclaro ejercicio de equilibrio de condiciones sociales, políticas y económicas, o lo que es también conocido como glocalización (Acosta, 2003). Esto último, basado en el reconocimiento de una lógica que impone regulaciones homologadoras que, si bien es cierto, apuntan a temas de oportunidades de estudio, titulación, movilidad académica y empleabilidad, no es menos cierto que desconocen las condiciones sociales, económicas y políticas en los que se hallan circunscritos los sistemas educativos nacionales. Así lo destacan, por cierto, investigadores como Leal, Barreto y Oregioni (2020) y Sperduti (2017), al apuntar que los modelos internacionalización desarrollados en América Latina no pueden seguir anclándose en relacionamientos desiguales con lugares hegemónicos en la producción de conocimiento en otros lugares del mundo, modelos en los que se sigan desconociendo realidades, ampliando brechas y desigualdades, concretando relaciones de dependencia en el ámbito del dominio saber-poder, ensanchando disparidades y enfatizando enfoques neoliberales, lo que se conoce también como capitalismo académico. Por lo tanto, las instituciones universitarias latinoamericanas tienen un desafío mayúsculo en este contexto.

Si hablamos de desafíos, tenemos que, la disminución cuantitativa en términos de movilidad académica estudiantil, es un tema que se ha destacado en otras investigaciones como una de las dimensiones más afectadas por la pandemia en cuanto a internacionalización se refiere $(\mathrm{CRUCH}$, 2020; Taşçi, 2021). Y esto, no sólo en términos de salubridad, sino también por la afectación económica de las instituciones. En vista de ello, trabajos como los de Taşçi (2021) y Bruhn (2020), suponen que las estrategias de internacionalización tienen que apuntar a la virtualidad (en diferentes formas) como mecanismo de apoyo en relación con la movilidad académica de estudiantes. Eso ha sucedido en la UnACh justo en 2020, y, de hecho, se ha destacado el incremento de actividades y participación de estudiantes en este tipo de instancias, tal y como ha sucedido también en todo Chile (CRUCH, 2020). Las facilidades que ofrece la virtualidad han permitido el acomodo y la adaptación en las formas tradicionales de asistencia presencial a formas de asistencia y participación virtual, lo que ha generado, además, el desarrollo de modalidades híbridas que, sin duda alguna, modificarán la forma de entender los procesos didácticos (Moreno, 2021; Rama, 2021).

Ahora bien, los procesos de articulación abarcan diversas dimensiones del ámbito universitario en las que se produce un impacto considerable en una variedad de funciones, en distintos niveles, actividades, participantes y recursos. Esta investigación permite constatar que la UnACh ha venido avanzando en estos aspectos, no sólo en relación con la cantidad de actividades, sino también con 
otros elementos de importancia e interés para la universidad. Es posible advertir que en años como el 2017 y el 2018, fueron pocas las instancias de internacionalización, además de estar reducidas en función de acciones de intercambio de docentes y estudiantes. El año 2019 presenta un leve crecimiento, sin embargo, el año 2020 destaca por ser el año en el que se han superado las expectativas y todos los indicadores referidos a la cantidad de actividades, cantidad de estudiantes participantes, profesores participantes, colaboración de investigaciones, además de diversificar las instancias de articulación interuniversitaria. Ha sido puesto en escena que la pandemia y el confinamiento impactan las formas $\mathrm{y}$ modalidades en las que se pueda estar operando procesos de internacionalización, confirmándose lo que ya ha venido siendo advertido por investigadores como Blanco (2021). Puede que la consolidación de estos esfuerzos se vean amenazados por la pandemia y medidas de confinamiento en función de la necesidad de presencialidad en algunos casos, no obstante, se hace preciso considerar que, así como se han generado múltiples alternativas para la docencia y la investigación, también se han generado alternativas para que se den instancias de internacionalización. Es decir, la virtualidad aceleró procesos, agilizó otros y permitió mayores facilidades para el desarrollo de acciones conjuntas entre instituciones, además de minimizar el impacto financiero que esto pudo haber tenido en otro contexto.

Adaptación, parece ser lo que viene acompañado de estos tiempos en pandemia, y esto ha significado, poniendo un ejemplo entre varios casos, la inversión financiera y de tiempo para la formación del personal docente, administrativo y de estudiantes en modalidades virtuales, ha implicado también la adquisición de software y plataformas virtuales, ha significado la ampliación de red de internet y aumento de la capacidad de transmisión, ha significado toda una adaptación a nivel curricular, de metodologías de enseñanza, de la evaluación, etc. Así lo ha destacado el Ministerio de Educación (2020), generando un marco amplio para el desarrollo de iniciativas institucionales en la educación universitaria. Todo este esfuerzo ha sido necesario, ya que la internacionalización implica también que quienes interactúan posean un mínimo de condiciones para entablar esfuerzos y articular con otros contextos, personas e instituciones. Esto ha sido avizorado también en una investigación realizada por Parker y López (2020), a partir del análisis de la realidad en la Universidad de Santiago de Chile, en la que, no sólo se ha advertido el crecimiento de la virtualidad para la internacionalización, sino que también ello implica el necesario ajuste a formas de internacionalización postpandemia. Es decir, sostienen que, independientemente de la superación de la pandemia y de la vuelta a la presencialidad, el proceso de internacionalización, tendrá una cuota importante en relación con la virtualidad.

En otro orden ideas, tenemos que, la mayor participación de docentes ha sido mayor que la de estudiantes en la 
UnACh, debido a que esta participación se ha dado a partir de instancias de colaboración en proyectos de investigación en conjunto con otras instituciones, la vinculación de los docentes a grupos de investigación, la participación de docentes en congresos internacionales como invitados de instituciones con las que se tienen convenios, entre otros. Las actividades en las que se suman los estudiantes son aun incipientes, dada la poca experiencia que ha tenido la UnACh en términos de movilidad estudiantil, pero sí va tendiendo a una mayor colaboración e intercambio (movilidad académica) a cuenta de convenios interinstitucionales con instituciones de la red adventista de universidades, y al mismo tiempo con instituciones universitarias no adventistas con las que hay convenios de colaboración estrechos. Un caso en este contexto es el de la Universidad de Cundinamarca, Colombia, con la que se han generado intercambios estudiantiles, y con trabajo colaborativo a partir de proyectos de investigación, además de aulas espejo en varias asignaturas (UnACh, 2020a, 2020b, 2020c).

En un trabajo desarrollado por Contreras (2015), se advierte que las instituciones universitarias chilenas no podrían catalogarse como universidades internacionalizadas, sino como instituciones que llevan a cabo actividades de internacionalización, incluyendo en esta caracterización a las universidades más desarrolladas en Chile, y las que tienen mayor tradición e historia. En este sentido, puede inferirse, además, que las instituciones que generan procesos incipientes de internacionalización, van buscando oportunidades de desarrollo según van apareciendo en instituciones que tienen horizontes similares en dicho ámbito. $\mathrm{Y}$ otro de los elementos que demuestra lo incipiente de estos procesos, es precisamente la desproporción en cuanto a la participación de quienes finalmente son beneficiarios de dichas instancias de internacionalización. Por ello se hace necesaria la definición de una política clara y progresiva en torno a la internacionalización como proceso instalado en la academia. Y esta definición política implica una buena práctica que apunta a la consolidación de los procesos de internacionalización. Práctica que no puede reducirse a la sola definición, sino que debe ampliarse a la instalación y ejercicio de mecanismos de internacionalización que convoquen a una mayor cantidad de participantes y beneficiarios.

Para que un proceso de internacionalización pueda en realidad consolidarse, necesita ser abordado justamente desde la perspectiva de buenas prácticas, tal y como lo plantean en sus investigaciones, Llano et al. (2021) y Gacel-Ávila (2021). Una de estas prácticas apunta hacia la dinamización de la articulación a partir de la multidimensionalidad del proceso. Si bien es cierto, la UnACh es considerada una universidad pequeña al hacerse comparaciones con otras instituciones de mayor historia, tamaño y cobertura en Chile, también es cierto que ha emprendido esfuerzos asociados a la internacionalización, esfuerzos que implican una mirada en la diversidad, 
esto es, procesos que miran la articulación en docencia, investigación y vinculación; procesos que atienden la vinculación de estudiantes, docentes y demás funcionarios de la institución; procesos que se desarrollan con instituciones públicas y privadas; procesos que se desarrollan con instituciones latinoamericanas, norteamericanas, pero también de continentes como Europa y África. Sebastián (2011), al hablar de dimensiones de la internacionalización, plantea el esfuerzo interno que debe hacer la institución para acoplarse a una mínima común internacional, y al esfuerzo externo que finalmente se concreta en el ejercicio y la operatividad de convenios y demás relacionamientos. $\mathrm{Y}$, en el caso del estudio realizado en la UnACh, se advierten algunos mecanismos de avance en cuanto a internacionalización en focos de multidimensionalidad. Se destaca el señalamiento de una serie de indicadores que permitirían generar mínimos estándares internacionales a fin de mejorar las prácticas de internacionalización.

Ahora bien, un elemento de importancia radica en la necesidad de ajustar las instancias de internacionalización a los contextos, tal y como lo destacan Gómez (2020), Muñoz (2016) y Quiroga y Trujillo (2020) en sus investigaciones. La contextualización en ámbitos de internacionalización refiere a procesos de glocalización, tal y como lo señalan Acosta (2003) y más recientemente Blanco (2021), y esto se ha asociado a su vez, a procesos de calidad en instancias de internacionalización y acreditación, resaltados también por Ramírez-Valdivia et al. (2020) y Sebastián (2017). Tal elemento es relevante habida cuenta de que los resultados de esta investigación revelan que la UnACh considera propuestas, proyectos y convenios tributantes a la misión y visión institucional en función de las situaciones de las carreras y los distintos programas institucionales, sean de pregrado o de posgrado, atendiendo al contexto regional y nacional, e inscrito en el marco de un proyecto institucional propio. Además, esto puede advertirse en la investigación, en la vinculación con instituciones iberoamericanas, $\mathrm{y}$ especialmente latinoamericanas, de habla castellana, instituciones adventistas, y que cuenten con carreras y programas afines. Pero, sin caer en un ejercicio de provincianismo, también se han adelantado acciones, proyectos $\mathrm{y}$ convenios con instituciones europeas, norteamericanas, africanas, de habla inglesa, no confesionales, instituciones públicas y privadas, entre otras. Todo ello contextualizado en el marco de la integración regional y un sistema de relaciones basado en relaciones simétricas, tal y como es destacado y sugerido en investigaciones como la de Blanco (2021) y Perrota (2019).

Finalmente, vale la pena destacar que, en relación con el objetivo de esta investigación, se presentan dos elementos que son centrales. Uno de ellos radica en que las instancias de internacionalización en las que ha participado la UnACh, se han ido acrecentando a propósito de la virtualidad, aprovechándose la oportunidad 
que brindan las tecnologías de información y comunicación, lo que abre espacios y posibilidades múltiples, dado que esto otorga facilidades en términos de costos y presupuestos, en términos de tiempos y plazos, la movilidad académica $\mathrm{y}$ las disposiciones para toma de decisiones en acciones conjuntas. Por supuesto, habrá acciones que podrían ralentizarse en el marco de la pandemia y las medidas de confinamiento que son distintas en los Estados nacionales, dado que deben generarse de forma exclusiva en presencialidad, pero estas serían mínimas en comparación con lo que podría avanzarse en virtualidad, tal y como ha quedado demostrado en las instancias de internacionalización generadas en el año 2020, año en el que se ha dado el punto más alto de internacionalización de la institución. Por otro lado, está el tema de que las acciones alcanzadas en función de internacionalización cuentan con altísimas probabilidades de continuidad, lo que podría generar un cúmulo importante de acciones que consolidarán la academia en las dimensiones en las que la universidad viene generando este tipo de esfuerzos, a saber: docencia, investigación y vinculación con el medio, y siendo más concretos, en el resultado de dichas instancias de internacionalización, como lo son los proyectos de colaboración académica, trabajo en redes, investigación inter-universidades, pasantías estudiantiles, entre otros. Claro está, tanto la virtualidad como la continuidad de los programas de internacionalización, son variables que dependen de los mecanismos que logre agenciar la institución para consolidar lo alcanzado, tal y como lo declara Gacel-Ávila (2021) haciendo referencia a las buenas prácticas de internacionalización.

\section{Conclusiones}

La pandemia ha tenido una incidencia, si se quiere, positiva, en cuanto al tema de la internacionalización en la UnACh, ya que ha permitido que docentes, estudiantes y funcionarios administrativos de diferentes carreras, programas, facultades y unidades de la UnACh, participen de instancias académicas internacionales que probablemente en presencialidad no habrían sido posibles, en términos de frecuencia y participación cuantificada. Esto se ha destacado en el incremento superlativo de las instancias de internacionalización en el año 2020 en relación con los demás años considerados en el estudio. Este proceso se ha visto ampliamente favorecido por las facilidades que reporta la virtualidad, insertando a la UnACh en una tendencia importante asociada al crecimiento de instancias de internacionalización a partir de las tecnologías de información y comunicación.

La UnACh ha generado procesos de internacionalización que apuntan hacia la multidimensionalidad de estos; además, se ha abierto a una cantidad importante de acciones, proyectos, modalidades de cooperación, con instituciones de varios países, siendo Latinoamérica la región con la que mayor relacionamiento y vinculación se ha alcanzado, ello, sin cerrarse a experiencias de otras latitudes como Europa, Norteamérica y África. 
Si bien es cierto, hay un cúmulo de actividades de internacionalización generadas en el contexto de convenios, $y$, dado que hay un umbral de actividades y procesos que se desarrollan sin estar enmarcados en convenios interinstitucionales, se hace preciso consolidar redes de vinculación académica tanto para docentes como para estudiantes, así como la generación de nuevas instancias de articulación de otros funcionarios de la institución, a partir de convenios que permitan sentar las bases de una cooperación mucho más sostenida y sistemática en el tiempo, brindando nuevas oportunidades a las instituciones en cuestión.

Las instancias de internacionalización generadas en el ámbito del período en estudio, son instancias que se corresponden con las necesidades formativas e institucionales de la universidad, dado que provienen de los intereses de las mismas carreras, programas de posgrado, facultades $y$ direcciones académicas, encontrando sus correlatos en las instituciones con las que se tienen los convenios.

Los esfuerzos en desarrollo avizoran posibilidades de continuidad y ello avista términos posibles para convenios y el desarrollo de mecanismos de internacionalización en tiempos de postpandemia. Finalmente, se hace preciso generar un proceso de sistematización de información mucho más preciso, dado que se dieron muchas actividades que al ser declaradas, no se informó la unidad participante. Al igual que, la declaración de este tipo de instancias debe ser obligatoria en la institución, por cuanto opera en términos de relaciones interinstitucionales y de representación universitaria.

\section{Contribución de autores}

N.G.L. Idea, revisión de literatura, metodología, análisis de datos, redacción y revisión final del artículo.

A.D.R.R. Metodología, análisis de datos, redacción y revisión final del artículo.

M.C.A. Metodología, análisis de datos, redacción y revisión final del artículo.

M.C.N. Metodología, análisis de datos, redacción y revisión final del artículo.

\section{Referencias}

Abba, M. J., y Streck, D. R. (2021). The 1918 Córdoba Reform and University Internationalization in Latin America. Revista História da Educação, 25(e102256), 1-32. http://dx.doi.org/10.1590/2236-3459/102256

Acosta, A. (2003). En la encrucijada de la glocalización. Algunas reflexiones desde el ámbito local, nacional global. Polis, 1(4), 1-19.

Albornoz, O. (2013). La universidad ¿reforma o experimento? Venezuela: IESALC-UNESCO.

Albornoz, O. (2018). Mitos, tabúes y realidades de las universidades. Volumen I. Cambios en las sociedades, reformas en las universidades. Venezuela: IESALC-UNESCO \& Universidad Central de Venezuela.

Bermejo, J. (2016). Ideologías en conflicto en el siglo XXI: islamofobia vs occidentalofobia. Revista de paz y conflictos, 9(1), 133-156. 
Blanco, G. (2021). El reto de adaptar los consorcios y las redes universitarias a la nueva normalidad. Revista de Educación Superior en América Latina, (10), 43-46.

Bruhn, E. (2020). Virtual Internationalization in Higher Education. Wbv Media GmbH \& Co. KG. https://doi.org/10.3278/6004797w

Buckner, E., Clerk, S., Marroquín, A., y Zhang, Y. (2020). Strategic Benefits, Symbolic Commitments: How Canadian Colleges and Universities Frame Internationalization. Canadian Journal of Higher Education, 50(4), 20-36.

Caamaño, C., y Chacón, F. (2017). Internacionalización neoliberal de la Educación Superior Pública. Transformación educativa, sustentabilidad y prácticas emancipatorias. Repositorio Institucional de la Universidad de Costa Rica.

Cancino, R. (2010). El modelo neoliberal y la educación universitaria en Latinoamérica. El caso de la universidad chilena. Sociedad y Discurso, (18), 149-165.

Consejo de Rectores de las Universidades ChilenasCRUCH. (2020). Internacionalización en contexto de crisis: nuevos lineamientos y desafios para las universidades del consejo de rectores de Chile. Chile: Del autor.

Contreras, P. (2015). Conceptualización y experiencia de la internacionalización del pregrado chileno. Calidad en la Educación, (43), 169-200.

De Wit, H., y Hunter, F. (2015). El futuro de la internacionalización de la educación superior en Europa. International Higher Education, (83), 2-4.

Díaz-Castrillón, F., y Toro-Montoya, A. (2020). SARS-CoV-2/COVID-19: el virus, la enfermedad y la pandemia. Medicina \& Laboratorio, 24(3), 183-205. https://doi. org/10.36384/01232576.268
Dirección de Docencia (2021). Informe de actividades de internacionalización. Chile: Universidad Adventista de Chile.

Escudero, J. C. (2010). Educación superior en Chile y su internacionalización. Revista HISTEDBR On-line, 10(38), 12-23. https: //doi.org/10.20396/rho.v10i38.8639686

Fairlie, A., Portocarrero, J., y Herrera, E. (2021). Desafíos de digitalización para la internacionalización de la educación superior en los países de la Comunidad Andina. Documentos de Trabajo Fundación Carolina, (46), 1-24.

Gacel-Ávila, J. (2020). COVID-19: Riesgos y oportunidades para la internacionalización de la educación superior en México. Revista de Educación Superior en América Latina, (8), 37-40.

Gacel-Ávila, J. (2021). The Importance of Internationalization Today and the Leadership Role of IAU. En: Van't Land, H., Corcoran, A., Iancu, D-C. (Eds). The Promise of Higher Education. Essays in Honour of 70 years of IAU, pp. 89-94. Springer: Cham, Switzerland. https:// doi.org/10.1007/978-3-030-67245-4_14

García, C. (2005). Complejidades de la globalización e internacionalización de la educación superior: Interrogantes para América Latina. Cuadernos del CENDES, 22(58), $1-22$.

Giroux, H. (2020). La pandemia de Covid-19 está exponiendo la plaga del Neoliberalismo. Praxis Educativa, 24(2), 1-13. https://doi. org/10.19137/praxiseducativa-2020-2402 02

Gómez, P. (2020). ¿Qué sucederá con la internacionalización de la educación superior y la movilidad estudiantil después del COVID-19? Revista Digital de Investigación en Docencia Universitaria, 14(2). https://doi.org/ 10.19083/ridu.2020.1348 
Grosfoguel, R. (2014). Las múltiples caras de la islamofobia. De Raiz Diversa, 1(1), 83-114.

Kotorov, I., Krasylnykova, Y., Zhdanov, P., y Mazarra, M. (2020). Finding the right understanding: Twenty-first century university, globalization and internationalization. In: Bruel, J-M., Capozucca, A., Mazzara, M., Meyer, B., Naumchev, A. y Sadovykh, A. (Eds.). Frontiers in software engineering education. (pp. 341-353). Springer.

Latorre, P., y Ramírez, M. (2021). Motivaciones y capacidades institucionales para la internacionalización de las universidades chilenas. Avanzando hacia la internacionalización integral. Revista de Educación Superior en América Latina, (10), 52-57.

Leal, F., Barreto, M., y Oregioni, M. S. (2020). Questionando o discurso e a prática de internacionalização da educação superior predominantes na América Latina. Arquivos Analíticos de Políticas Educativas, 28, 132. https://doi.org/ 10.14507/epaa.28.3904

Lemaitre, M. J. (2005). La calidad colonizada: universidad y globalización. Revista de la Educación Superior, XXXIV(133), 123-134.

León R., y Madera, L. (2016). La internacionalización universitaria, un imperativo de la educación superior en el contexto latinoamericano actual. Revista Encuentros, 14(2), 43-59. https://doi.org/ $10.15665 /$ re.v14i2.779

Llano, G., Carlozama, J., Tipán, D, y Tipán, G. (2021). La internacionalización en los procesos universitarios: un análisis estructural para universidades de Latinoamérica. Mendive, Revista de Educación, 19(1), 6-15.
Maas, A. (2018, 26 de noviembre). Las estrategias de las casas de estudio para atraer alumnos. NexNews Prensa escrita.

Ministerio de Educación. (2020). Plan de acción MINEDUC para Instituciones de Educación Superior. Chile: Del autor.

Mönckeberg, M. O. (2014). El negocio de las universidades en Chile, 2 ed. Chile: DeBolsillo.

Moreno, C. (2021). Reto de las universidades públicas durante la pandemia. En Jornada de Conferencias Apertura del Ciclo 2021 posgrados. Ponencia presentada en el Centro Universitario de Tonalá, Universidad de Guadalajara, México, 4 de marzo de 2021.

Munadi, M. (2020). Systematizing Internationalization Policy of Higher Education in State Islamic Universities. International Journal of Higher Education, 9(6), 96-106. https://doi.org/10.5430/ijhe.v9n6p96

Muñoz, L. (2016). Internacionalización de la educación superior: Una exploración introductoria acerca de su presencia en las carreras de posgrado de la Universidad de Costa Rica. Educación, 40(2), 1-28. http://dx.doi.org/10.15517/revedu.v40i2. 17214

Murillo, F., y Duk, C. (2020). El Covid-19 y las brechas educativas. Revista Latinoamericana de Educación Inclusiva, 14(1), 11-13. http://dx.doi.org/10.4067/S0718-737820 20000100011

Ordorika, I. (2020). Pandemia y educación superior. Revista de la Educación Superior 194, 49, 1-8.

Organización de las Naciones Unidas para la Educación, la Ciencia y la Cultura -UNESCO, e, Instituto Internacional para la Educación Superior en América Latina y el Caribe - IESALC (2020). COVID-19 
y educación superior: de los efectos inmediatos al día después. Análisis de impactos, respuestas políticas y recomendaciones. Recuperado de: https://cutt.ly/JEuyQKy

Otzen, T., y Manterola, C. (2017). Técnicas de muestreo sobre una población de estudio. International Journal of Morphology, 35(1), 227-232. http://dx.doi.org/10.40 67/S0717-95022017000100037

Parker, C., y López, F. (2020). Prospectiva y consecuencias del COVID-19, y su impacto en la Educación Superior. Documento de Trabajo, Edición PrePrint, Vicerrectoría de Postgrado, Universidad de Santiago de Chile.

Perrota, D. (2019). Didou Apetit, S. (2017). La internacionalización de la educación superior en América Latina: transitar de lo exógeno a lo endógeno. México: Unión de Universidades de América Latina y el Caribe. Integración y Conocimiento, 8(1), 137-139.

Quiroga, S., y Trujillo, H. (2020). Internacionalización universitaria: diversidad y desafíos en América Latina. En el $12^{\circ}$. Congreso Internacional de Educación Superior Universidad 2020. Ponencia presentada en La Habana, Cuba, 10 -14 de Febrero de 2020.

Rama, C. (2021). La nueva educación híbrida. México: Cuadernos de Universidades 11.

Ramírez-Valdivia, M. T., Latorre, P., y Bustos, J. M. (2020). ¿La calidad como motor de la internacionalización de las universidades chilenas? En 4th International Conference on Quality Engineering and Management. Ponencia presentada en Universidad of Mhino, Braga- Portugal, 21-22 de septiembre de 2020.

Reyes, A. (2016). Educar. ¡La clave!, ;El riesgo! Venezuela: Fundación Editorial El Perro y la Rana \& Ediciones UNEY.
Santos, A., y Román, M. (2003). Evolución de la internacionalización de la Facultad de Ciencias de la Conducta. Tiempo de Educar, 4(7), 13-52.

Sebastián, J. (2005). La internacionalización de las universidades como estrategia para el desarrollo institucional. Innovación Educativa, 5(26), 1-10.

Sebastián, J. (2011). Dimensiones y métrica de la internacionalización de las universidades. Universidades, (51), 3-16.

Sebastián, J. (2017). Algunos dilemas en torno a la internacionalización de la educación superior. Educación Superior y Sociedad, 21, 119-145.

Sperduti, V. (2017). Internationalization as Westernization in Higher Education. Comparative \& International Education, 9, 9-12.

Taşçi, G. (2021). The impact of COVID-19 on Higher Education: Rethinking internationalization behind the iceberg. International Journal of Curriculum and Instruction, 13(1), 522-536.

Universidad Adventista de Chile - UnACh. (2018). Informe de acreditación institucional. Chile: Del autor.

Universidad Adventista de Chile - UnACh. (2020a). UNACH recibe a estudiantes de intercambio de la Universidad de Cundinamarca, Colombia. Recuperado de: https://www.unach.cl/2020/03/unachrecibe-a-estudiantes-de-intercambio-de-1 a-universidad-de-cundinamarca-colombia/

Universidad Adventista de Chile - UnACh. (2020b). Facultad de Ingeniería y Negocios de la UNACH realiza Primer Aula Espejo con la Universidad de Cundinamarca, Colombia. Recuperado de: https://www. unach.cl/2020/06/unach-realiza-primer-a ula-espejo-con-la-universidad-de-cundin amarca/ 
Universidad Adventista de Chile - UnACh. (2020c).

Facultad de Educación y Ciencias Sociales realiza Aula Espejo Virtual con la Universidad de Cundinamarca, Colombia. Recuperado de: https:// www.unach.cl/2020/06/facultad-de-educ acion-y-ciencias-sociales-realiza-aula-es pejo-virtual-con-la-universidad-de-cundi namarca-colombia/

Universidad Nacional Autónoma de Honduras UNAH. (2020). Protocolo de internacionalización en el marco de la pandemia SARS-COV-2.

Villalobos, C., y Quaresma, M. L. (2015). Sistema escolar chileno: características y consecuencias de un modelo orientado al mercado. Convergencia. Revista de Ciencias Sociales, (69), 63-84. https:// doi.org/10.29101/crcs.v22i69.3634 